\title{
The effect of surfactants on the dynamics of phase separation
}

\author{
Mohamed Laradji, Hong Guo, Martin Grant and Martin J Zuckermann \\ Centre for the Physics of Materials and Physics Department, Ernest Rutherford Building, \\ 3600 Rue University, McGill University, Montréal, Québec, Canada H3A 2T8
}

Received 7 April 1992

\begin{abstract}
The dynamics of phase separation in binary systems containing surfactants is investigated by means of a time-dependent Ginzburg-Landau model. The Langevin equations for two scalar fields are solved numerically in two spatial dimensions. One of these fields is the order parameter representing the local difference in the concentrations of the two phase-separating components and the second is the local surfactant concentration. Different conservation laws of the fields, which represent different models for the dynamics of phase separation, are investigated. In all models, the average domain size for intermediate to late times is characterized by anomalously slow dynamics caused by the accumulation of surfactants at the interfaces and by the concomitant decrease in the driving force. Although all models exhibit similar slow growth, the domain structure is found to depend strongly on the nature of the conservation law. Indeed, the structure factor exhibits a peak at $k>0$ when the order parameter is conserved, whereas the peak is found to lie at $k=0$ if the order parameter is not conserved. Finally, dynamical scaling in both real- and Fourier-space correlation functions for the order parameter is found during the intermediate time regime.
\end{abstract}

\section{Introduction}

Ternary mixtures containing surfactants exhibit a considerable number of fascinating microstructures such as emulsions, microemulsions, colloids and foams [1,2]. This is due to the fact that the head of a surfactant molecule interacts attractively with one component of the mixture while the tail also has an attractive interaction with a second component. The surfactants can therefore stabilize mixtures of two otherwise immiscible components by the formation of interfaces composed of surfactant films. Amphiphiles are typical surfactants: these molecules have hydrophilic polar heads and hydrophobic tails and can therefore form emulsions, microemulsions and colloids when mixed with water and oil, and foams when spread on an air-water interface. The structure of microemulsions can be described in terms of microdomains of a few hundred Angstroms correlated over short distances and separated by thin surfactant monolayers. When the concentrations of water and oil are comparable, these domains have a bicontinuous structure. The short-range order is manifested by a broad peak in the water-water scattering intensity at a non-zero value of the wave vector [3-5]. The position of this peak shifts towards larger values of the wave number as the concentration of surfactants increases, implying a decrease in the average domain size [3]. For unequal concentrations of water and oil, microemulsions are often composed of droplets of the minority component. A-B diblock copolymers also act like surfactants when mixed with a blend of $A$ and $B$ homopolymers and they can 
therefore produce stable phases $[6,7]$. The phase equilibria of systems containing surfactants has been examined in many experimental and theoretical studies and some of the latter are based on microscopic or coarse-grained theories [8-12]. In this work we study the dynamics of microphase separation in ternary mixtures containing surfactants. To this purpose we first give a summary of the dynamics of phase separation in binary alloys and fluids, which is reasonably well understood [13]. When binary systems are critically quenched from a high-temperature disordered state to a state inside the unstable coexistence region of the phase diagram, they evolve towards equilibrium via the formation of domains. This domain structure coarsens during intermediate to late times until complete phase separation takes place. The average domain size at late times obeys an algebraic growth law, $R(t) \sim t^{n}$, which has been observed in experiments and numerical studies of the Ising model and related models [14-16]. The numerical value of $n$ depends on a few physical constraints such as the presence or absence of conservation laws. In binary systems, the dynamics of phase separation is governed by the minimization of the surface energy of the domains. The driving force of the dynamics is proportional to the local curvature of domain walls in the case where the order parameter is non-conserved.

When surfactants are present in the binary mixture, the nature of the dynamics can change. This is because surfactants are interfacially active molecules and their solubility in water- or oil-rich regions is usually very low. They therefore diffuse towards the interfaces where they can simultaneously interact with water and oil. Now, at early times, the domains are quite small. The average interfacial surfactant concentration is also small, implying a relatively large driving force and then a fast coarsening of the domains. However, the interfacial concentration of surfactants increases with increasing time since this helps to screen the interaction between water and oil molecules, thus reducing interfacial free energy. As a result the local interfacial tension between water and oil regions decreases which then leads to a decrease in the driving force. The domain growth will furstly slow down and finally cease when the effective interfacial tension between the domains vanishes, corresponding to microphase separation.

Slowing down in domain growth has been observed in systems with quenched impurities [17, 18] or random fields [19-21] and in spin glasses [22]. In these systems the impurities pin the interfaces, thereby limiting the growth to logarithmically slow activated processes. Furthermore, the interfacial tension in these systems can be vanishingly small, leading to disordered equilibrium states as found for the twodimensional random field Ising model [23]. However, our surfactants cannot be regarded as quenched impurities since they are mobile and can diffuse rapidly, and so the phenomena, while related, have important differences.

Here we investigate the dynamics of phase separation for a binary mixture containing a small surfactant concentration using a Ginzburg-Landau model based on two local fields as described in section 2 . The first field is the order parameter of the system which represents the local difference in the concentrations of the two immiscible components and the second describes the local surfactant concentration. Two coupled Langevin equations for the space and time dependence of the two fields are then derived from the free energy functional and are solved numerically by iteration on two-dimensional grids over long periods of time. The results are given in section 3. Following Halperin et al [24], we solve the Langevin equations for models A, B, $C$ and $D$, which are defined by the presence or absence of conservation laws for the two fields. Here model D corresponds to the experimental situation for colloidal dis- 
persions, since both fields are conserved. In all four cases, we examine the effect of conservation laws on the dynamics as well as on the asymptotic microstructure of the system. Our results show a very slow growth during what is clearly a microphase separation, leading to an eventual saturation of the domain size which gives a structure with only short-range order. This behaviour was observed for all four models. The presence of surfactants therefore affects the growth rate and the average domain size drastically. Even though the domain growth saturates, the system was found to exhibit approximately self-similar behaviour as implied by the dynamic scaling behaviour of the real- and Fourier-space correlation functions at the intermediate time regime. A brief description of some of our results for model D was published elsewhere [25].

\section{Model and methods}

In this section we introduce the two-field Ginzburg-Landau free energy functional discussed above. Since water and oil are immiscible fluids, their equilibrium behaviour can be described by $\psi^{4}$ theory, where the order parameter, $\psi(x)$, represents the difference in the local concentrations of the two components. The simplest free energy which inciudes a third component whose local concentration is represented by a field $\rho(x)$, can be written as $\dagger$

$$
\mathcal{F}\{\psi, \rho\}=\int \mathrm{d} x\left[c(\nabla \psi)^{2}-r \psi^{2}+u \psi^{4}+g \rho^{2} \psi^{2}+a \rho^{2}-\mu \rho-\left(\mu_{\mathrm{w}}-\mu_{\mathrm{o}}\right) \psi\right] .
$$

This model was first introduced by Halperin et al [24] for critical dynamics with $\rho$ representing the local concentration of a third component, an energy density or an entropy density. Note that $c, r, u, g$ and $a$ are positive phenomenological constants for temperatures below the critical point, $T_{c}$. The parameter $r$ becomes negative above $T_{\mathrm{c}}$ and the two immiscible components can then mix in the absence of the third component. The fourth term in (1) guarantees that the local surfactant concentration (the $\rho$-field) remains small in the bulk where $\psi$ is large. Furthermore, a positive value of $a$ prevents the third component from forming clusters. $\mu$ is the chemical potential of the third component, and $\mu_{\mathrm{w}}$ and $\mu_{\mathrm{o}}$ are the chemical potentials for water and oil respectively. The equations of motion of the two fields can easily be derived from (1) and are given by

$$
\frac{\partial \psi}{\partial t}=-M_{\psi} \frac{\delta \mathcal{F}}{\delta \psi}+\eta_{\psi}(x, t)
$$

and

$$
\frac{\partial \rho}{\partial t}=-M_{\rho} \frac{\delta \mathcal{F}}{\delta \rho}+\eta_{\rho}(\boldsymbol{x}, t)
$$

where the thermal noise for both fields satisfies the fluctuation-dissipation relation

$$
\left\langle\eta(x, t) \eta\left(x^{\prime}, t^{\prime}\right)\right\}=2 T M \delta\left(x-x^{\prime}\right) \delta\left(t-t^{\prime}\right) .
$$

Here the Boltzmann constant has been set to unity, $T$ is the temperature and the kinetic coefficients $M_{\psi}$ and $M_{\rho}$ depend on the conservation laws and will be discussed 
later. Note that the model of (2) and (3) leads to complete phase separation, as discussed by Hohenberg et al [24,27], and belongs to the same universality class as the Ising model. Hence, during the dynamics of the phase separation, ordered domains will grow according to an algebraic law as previously stated [27, 28].

To study the effects of surfactants on phase separation, (1) needs to be modified such that $\rho$ are preferentially located at the interfaces. The detailed interaction of the surfactants with water and oil molecules, and their tendency to be adsorbed at interfaces, i.e., in regions with high gradients in $\psi$, is described to lowest order by the following term to be added to (1)

$$
\mathcal{F}_{\text {surfactant }}=-s \int \mathrm{d} x \rho(\nabla \psi)^{2}
$$

where $s$ is a positive constant. The physical properties of colloidal dispersions and emulsions are usually described in terms of a more complicated form of the freeenergy functional [12], but our main concern is to study the dynamics of microphase separation induced by the presence of surfactants for the simplest possible model. We will show that the addition of (5) to (1) causes the interfacial tension between unlike domains to vanish at very late times for non-zero concentrations of surfactants thereby inhibiting complete phase separation.

From (1) and (5), we can rewrite (2) and (3) as follows

$$
\begin{aligned}
\frac{\partial \psi}{\partial t}=-M_{\psi}[ & \left.-2 r \psi+4 u \psi^{3}+2 g \rho^{2} \psi-2(c-s \rho) \nabla^{2} \psi+2 s \nabla \rho \cdot \nabla \psi-\left(\mu_{\mathrm{w}}-\mu_{\mathrm{o}}\right)\right] \\
& +\eta_{\psi}(x, t)
\end{aligned}
$$

and

$$
\frac{\partial \rho}{\partial t}=-M_{\rho}\left[2 a \rho+2 g \rho \psi^{2}-s(\nabla \psi)^{2}-\mu\right]+\eta_{\rho}(x, t) .
$$

Following Halperin et al [24], we study the Langevin equations of (6) and (7) for models $A, B, C$ and $D$, which are defined by the presence or the absence of conservation laws. Both kinetic coefficients, $M_{\psi}$ and $M_{p}$, are constants for model A, implying the absence of conservation laws for both fields. For model B, $\psi$ is conserved by taking $M_{\psi} \propto-\nabla^{2}$, but $\rho$ is not conserved. For model C, $\psi$ is not conserved but $\rho$ is conserved so that $M_{\rho} \propto-\nabla^{2}$. Finally, in model $\mathrm{D}$, both fields are conserved. The last model corresponds to the usual experimental situation for colloidal dispersions since the concentrations of the various components are generally conserved. However, the other three models are useful for the study of the effect of conservation laws on dynamics. Model C, for example, can be used to describe the dynamics of a water-vapour interface occupied by surfactants. It should be noted that, in the absence of surfactants $s=0$, a temperature quench is followed by algebraic growth with the exponent $n=1 / 2$ for models $A$ and $C$, while $n=1 / 3$ for models $B$ and D.

Equations (7) and (8) are solved numerically on two-dimensional grids using Euler's method and a finite difference technique. Periodic boundary conditions are imposed in order to reduce finite size effects. However, it is important to note that the real dynamics of water and oil containing surfactants is not completely described by these two equations, since such systems are fluids and, therefore, hydrodynamic 
effects are expected to play some role. Our assumption is therefore valid only if the velocity field is overdamped. Binary mixtures of $A$ and $B$ homopolymers containing A-B diblock copolymers would therefore be good candidates for model D due to the low diffusion of polymers. Moreover, since we only study the two-dimensional case, which could well correspond to the case of a water-oil mixture containing a small amount of surfactant constrained between two plates separated by a very small distance, the convective mode is usually overdamped due to friction between the fluid and the plates.

The sample used in these simulations is a $128 \times 128$ square grid with a spatial mesh size of $\Delta x=0.7$. A large number of independent runs (usually more than 50) was made for all simulations. The values of parameters used in our simulations were $c=1 / 4, r=1 / 2, u=1 / 4, g=5 / 2, a=1 / 4$ and $s=1 / 4$, though the results are not sensitive to this particular choice. It is important to note that chemical potentials become ineffective in (6) and (7) if the corresponding Langevin equation is conserved; in this case the concentration of the relevant component is maintained at its initial value.

In this study we only examine the case where concentrations of water and oil are equal, implying that $\mu_{\mathrm{w}}=\mu_{0}$ in models $\mathrm{A}$ and $\mathrm{C}$, or the average initial value $\psi_{0}=0$ in models B and D. For simplicity, the thermal noises in (7) and (8) were taken to be zero since the inclusion of a finite noise should not change the dynamics significantly [15].

Domain growth was studied by calculating the real space pair-correlation function for the order parameter

$$
g(x, t)=\left\langle\left[\psi(x, t)-\psi_{0}\right]\left[\psi(0, t)-\psi_{0}\right]\right\rangle
$$

and its Fourier transforms, i.e., the structure factor

$$
S(k, t)=\left\langle\frac{1}{L^{2}}\left|\sum_{\boldsymbol{x}_{i}}\left[\psi\left(\boldsymbol{x}_{i}, t\right)-\psi_{0}\right] \mathrm{e}^{\mathrm{i} k \cdot \boldsymbol{x}_{i}}\right|^{2}\right\rangle .
$$

The same definitions hold for the $\rho$ field. The wave number, $k$, is defined as follows:

$$
k^{2}=\frac{2}{\Delta x^{2}}\left[2-\cos \left(k_{x} \Delta x\right)-\cos \left(k_{y} \Delta x\right)\right]
$$

where $k_{x}=(2 \pi / L) m, k_{y}=(2 \pi / L) n$ in units of $\Delta x$ and $m, n=0,1,2, \ldots, L$, where $L$ is the linear system size. This definition of the wave number arises from the use of the discrete Laplacian in two dimensions. The results in section 3 are presented in terms of the circularly averaged structure factor $S(k, t)=\sum^{\prime} S(k, t) / \sum^{\prime} 1$. Here $\Sigma^{\prime}$ denotes a sum over a circular shell defined by $n-\frac{1}{2} \leqslant k L /(2 \pi) \leqslant n+\frac{1}{2}$. The average domain size is obtained as a function of time from the second moment of $S(k, t)$

$$
R(t)=2 \pi\left(\sum_{k=0}^{k_{c}} S(k, t) / \sum_{k=0}^{k_{c}} k^{2} S(k, t)\right)^{1 / 2}
$$




\section{Results and discussion}

We first show results for model $D$ when both fields are conserved. A time mesh size $\Delta \tau=0.02$ was chosen for this case and it was found that smaller spatial or time mesh sizes gave essentially the same results. The kinetic coefficients for both fields were set to $1 / 2$. The final configurations found after 100000 iterations are shown in figure 1. The surfactant concentrations investigated were $\rho_{0}=0.1,0.15,0.17$ and 0.2 . The left column of figure 1 shows snapshots giving the spatial dependence of the order parameter, $\psi$, and the right column displays the spatial distribution of the local surfactant concentration, $\rho$. It is clear from figure 1 that the surfactants accumulate at the interfaces where they form thin layers, as expected from (5). The profile of the two fields along one axis is displayed in figure 2. Figures 1 and 2 show that the value of the $\rho$ field is extremely small in the bulk due to the fourth and fifth terms in the free-energy functional of (1). In contrast, the profile has strong arrow peaks at the interface. This implies that the system reaches a state in which the presence of order parameter gradients at the interfaces has no significant cost in free energy. Figure 1 shows that the number of interfaces increases with increasing surfactant concentration, $\rho_{0}$, leading to a reduction in the average domain size. This behaviour is usually observed in microemulsions for equal water and oil concentrations [3, 10]. Figure 1 also shows the interconnected nature of the domain structure for equal concentrations of water and oil, ie. $\langle\psi\rangle=0$. Closed domains (or micelles) are found when the concentrations of water and oil are considerably different from each other.

The average domain size, $R(t)$, was calculated from the circularly averaged structure factor using (11) and is shown in figure 3 as a function of time for several values of the average surfactant concentration, $\rho_{0}$. We observed fast transient behaviour at early times followed by slow dynamics, this being more pronounced for large values of $\rho_{0}$ and late times. $R(t)$ is displayed as a function of $\ln t$ in figure 4 . The observation that the domain growth is relatively fast at early times can be understood as follows. At these times, $R(t)$ is quite small, and therefore the proportion of interface is very large. This implies that the average concentration of surfactants at the interfaces is small and consequently that the interfacial tension is large leading to a strong driving force. Figure 4 shows that the time dependence of $R(t)$ at intermediate times is not a power law but is possibly logarithmic. The reason for such a slow growth is that the surfactants accumulate at the interfaces causing a considerable reduction in the interfacial tension and hence in the driving force. Microscopically, this occurs when the surfactants screen the two-body interactions between water and oil molecules. The domain growth finally becomes slower than logarithmic at later times, when the domain size approaches saturation and the interfacial tension tends to zero.

In model $\mathrm{C}$, the local surfactant concentration, $\rho$, is conserved, but the order parameter $\psi$ is not. In this case, the mobility of the order parameter and the kinetic coefficient of the $\rho$ field were both given values of $1 / 2$ and the size of the time mesh, $\Delta \tau$, was taken to be 0.002 . Figure 5 gives the final configurations for three surfactant concentrations $\rho_{0}=0.1,0.15$ and 0.2 . This figure shows that $R(t)$ decreases as the surfactant concentration increases and that surfactants accumulate at the interfaces as found for model D. However, the interconnected domains are structured differently from model D: they appear to be less correlated and to have a structure similar to that observed for the kinetic Ising model. This is due to the non-conservation of the order parameter, and will be discussed below in terms of the behaviour of the relevant structure factor. 

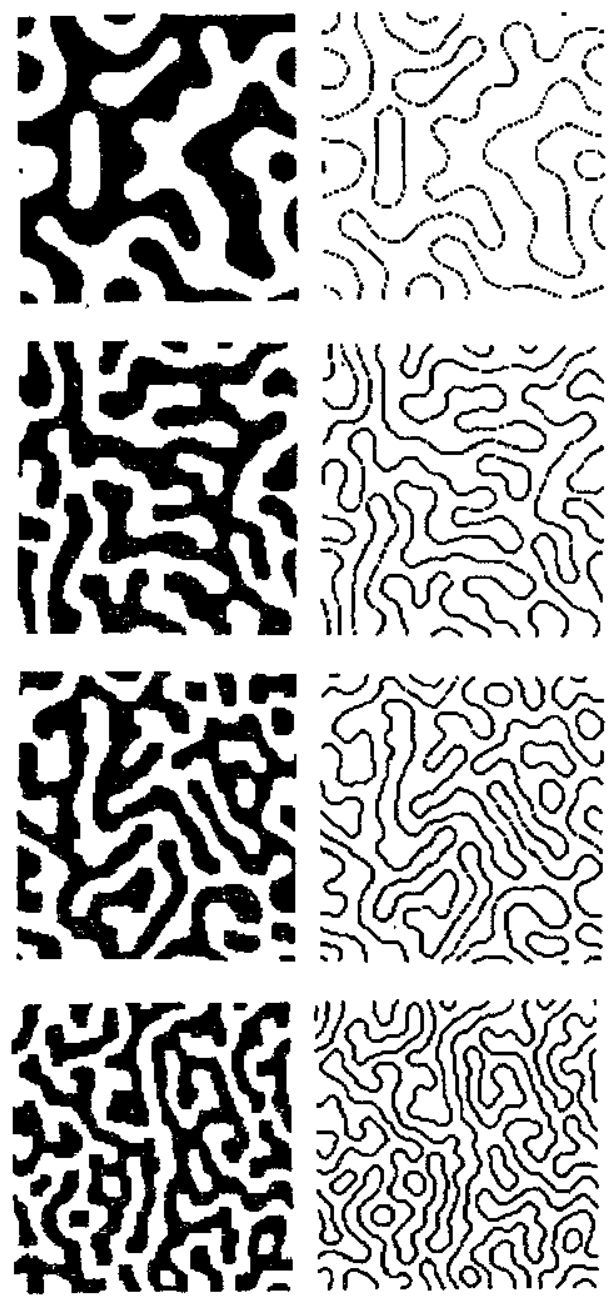

Figure 1. Final configurations (after 100000 iterations) in the case of model $D$ for values of $\rho_{0}=0.1$, $0.15,0.17$ and 0.2 , from top to bottom. The left column displays the spatial distribution of the $\psi$ field, in which black regions correspond to positive $\psi$, and white regions correspond to negative $\psi$. The right column shows the spatial distribution of the $\rho$ field. White regions correspond to small values if $\rho$, whereas black regions correspond to large values of $\rho$.

We also calculated $R(t)$ for model $\mathrm{C}$ from the second moment of the structure factor. This is shown in figure 6 for $\rho_{0}=0.17$. A relatively fast growth at early times, followed by extremely slow dynamics at late times is again observed. Similar behaviour was found for model $A$ where neither the order parameter nor the local surfactant concentration is conserved and the surfactant concentration is controlled by a chemical potential (see (7)). Figure 7 gives the final configuration for model $\mathrm{A}$ when $\mu=0.3$ corresponding to $\langle\rho\rangle \approx 0.197$. The corresponding values of $R(t)$ are given in figure 8 . This figure shows that the time evolution of average domain growth for model $\mathrm{A}$ is basically the same as for models $\mathrm{C}$ and $\mathrm{D}$.

The structure factor is the Fourier transform of the pair-correlation function, and is directly proportional to the scattering intensity, which can be measured experimentally by neutron scattering in bicontinuous microemulsions. Figure 9 gives the time evolution of the structure factor for model $\mathrm{D}$ when $\rho_{0}=0.17$. This figure shows that the structure factor calculated at early times exhibits a small peak at large wave numbers, indicating the occurrence of correlated fluctuations in the order parameter with a length scale equal to the inverse of the maximum wave number $k_{\max }$. The height 


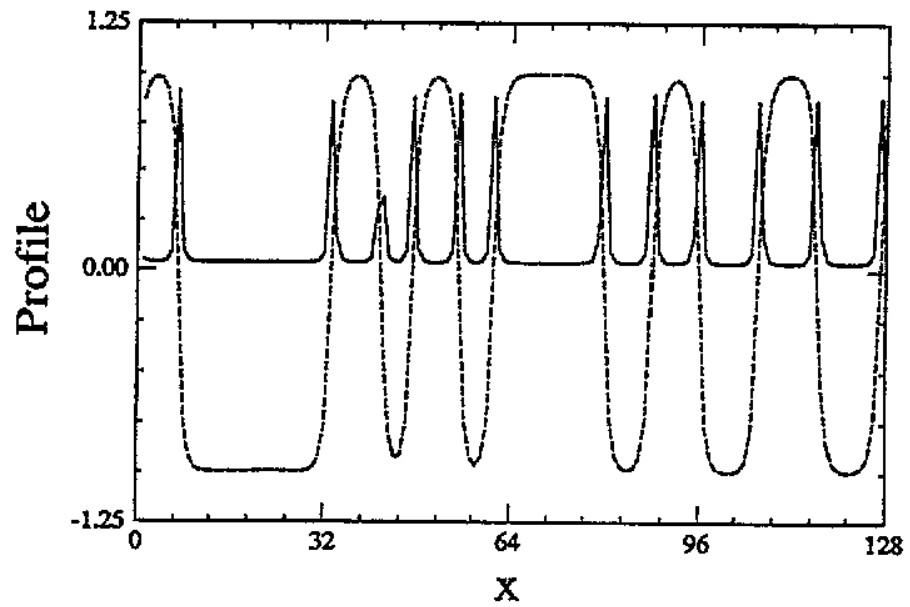

Figure 2. Profile along an axis of the two fields $\psi$ and $\rho$ at late times. (Model D, $\rho_{0}=0.15$.) The dashed line represents the $\psi$-field whereas the solid line represents the $\rho$-field.

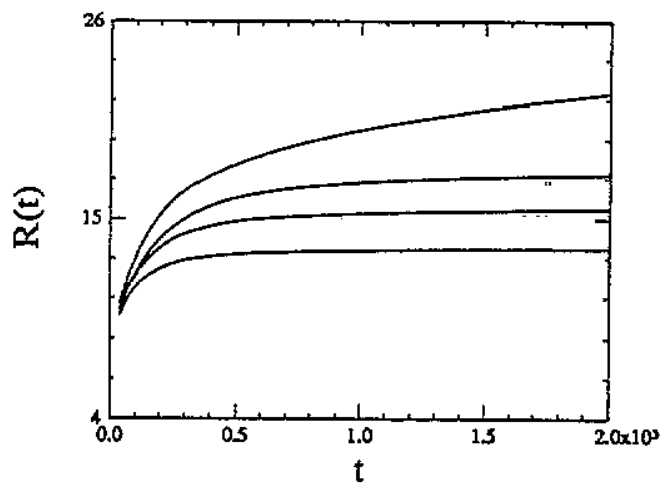

Figure 3. Time evolution of the domain size in model $\mathrm{D}$ for different values of $\rho_{0}=0.1,0.15$, 0.17 and 0.2 .

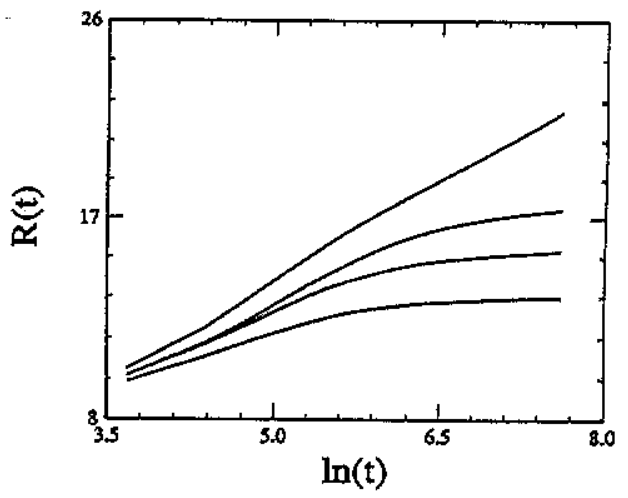

Figure 4. As in figure 3, but here $R(t)$ is plotted versus $\ln t$.

of the peak increases with increasing time, but $k_{\max }$ decreases implying a coarsening of the domain structure. At late times the peak hardly changes: this corresponds to a large interfacial concentration of surfactants. Figure 9 also shows that the structure factor becomes zero for $k=0$ since the order parameter is conserved. The same behaviour is observed for model B for which the order parameter is conserved and the $\rho$ field is not. The presence of a peak in the structure factor originates from the decaying oscillations in the pair correlation function due to correlation between domains as seen in figure 1 .

Figure 10 gives the structure factors for model $D$ corresponding to the final configurations for several surfactant concentrations. This figure shows that the height of the peak decreases and its position increases with increasing surfactant concentration due to the corresponding decrease in average domain size. Finally, we have checked 

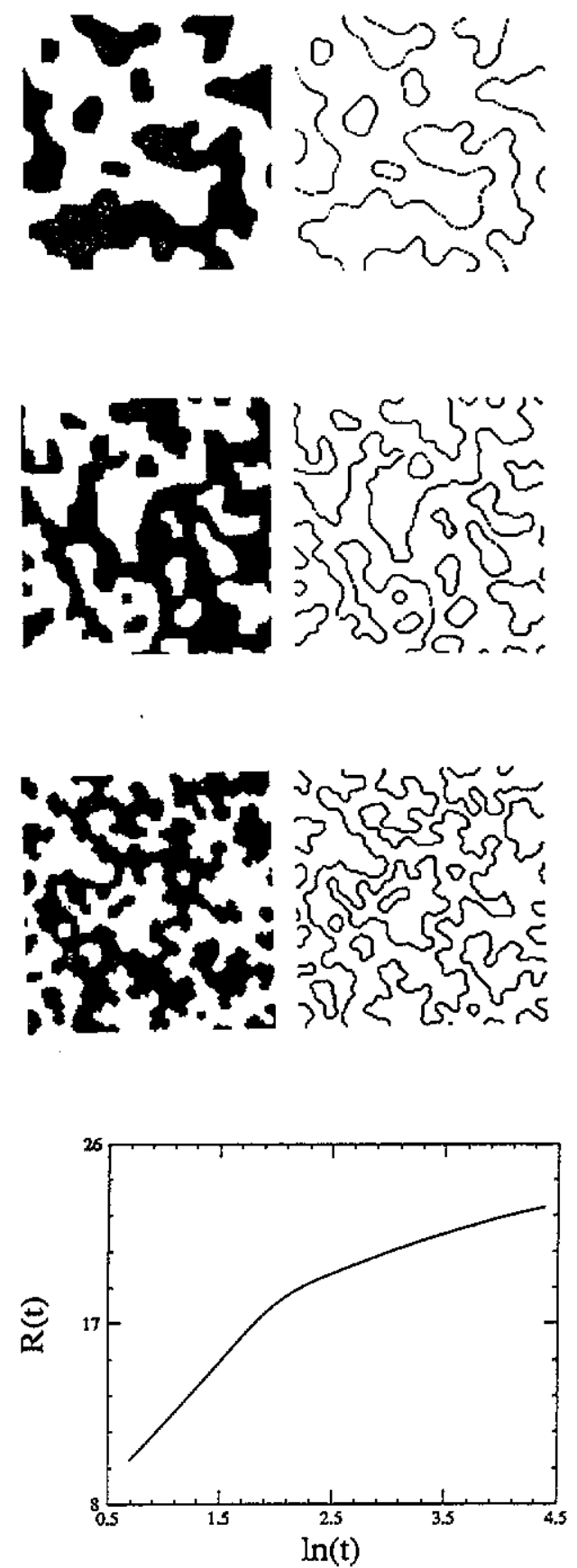

Figure 6. Time evolution of domain size for $p_{0}=$ 0.15 in the case of model $C$.
Figure 5. Final configurations (after 20000 iterations) in model $C$, for values of $\rho_{0}=0.1,0.15$ and 0.2. Details are as in figure 1 .

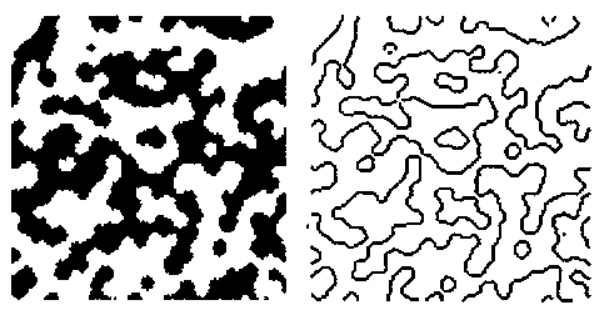

Figure 7. A final configuration (after 20000 iterations) for $\mu_{\mathrm{s}}=0.3$ in the case of model A. Details are as in figure 1.

that the Fourier transform of the surfactant density correlation function has a peak at approximately $2 k_{\max }$. This is consistent with the conjecture that the surfactant layers 


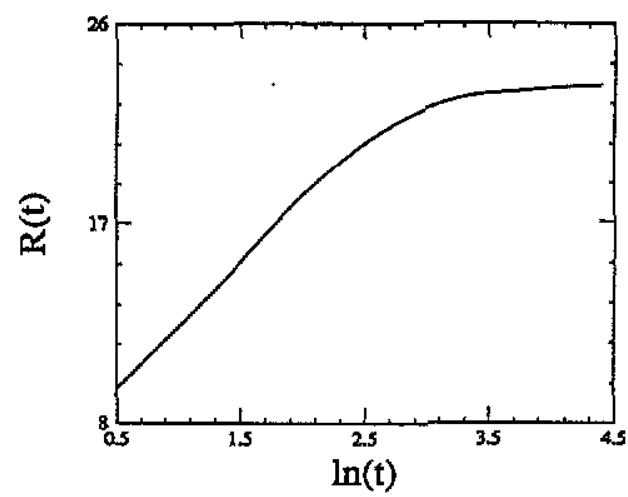

Figure 8. Time evolution of domain size for $\mu_{*}=$ 0.3 in the case of model $\mathrm{A}$.

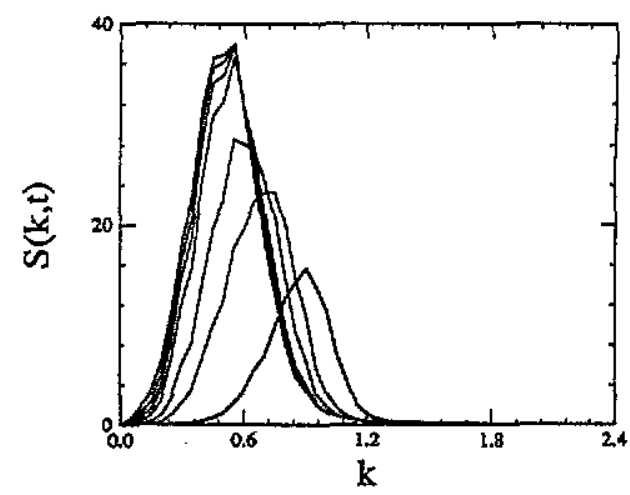

Figure 9. Time evolution of the structure factor in the case of model $D$ for $\rho_{0}=0.17$. Data correspond to times from $t=40$ up to $t=2000$.

are correlated with a length scale given by half the correlation length between water domains or between oil domains.

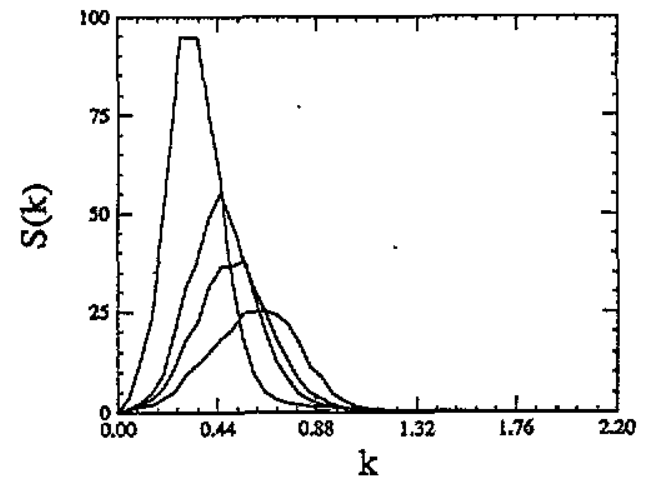

Figure 10. Final structure factors in model $D$ (after 100000 iterations). Curves from top to bottom correspond respectively to $\rho_{0}=0.1,0.15,0.17$ and 0.2 .

Binary systems undergoing phase separation often exhibit self-similar behaviour which is reflected in the dynamic scaling of the pair correlation function and the corresponding structure factor $[15,16]$. This occurs at late times when the average domain size, $R(t)$, becomes the only relevant length scale. For ternary systems containing surfactants, we have shown that $R(t)$ saturates at late times, implying that the width of the interface is not infinitely small compared with $R(t)$. However, since the interfacial width is still much smaller than $R(t)$, it is reasonable to make the usual scaling ansatz. This allows us to write the correlation function and the structure factor corresponding to the order parameter as follows

$$
G(r / R(t))=g(r, t)
$$

and

$$
F(x)=\left[1 / R(t)^{d}\right] S(k, t)
$$




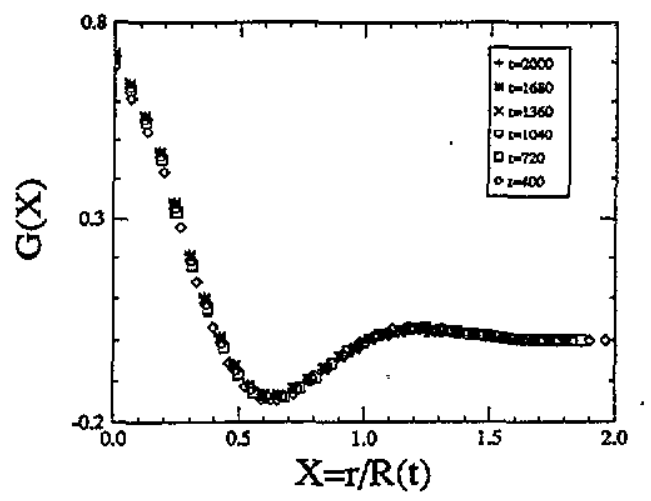

Figure 11. The scaled real-space correlation function in the case of model $D$ for $\rho_{0}=0.17$ is displayed at times ranging from $t=400$ to $t=2000$.

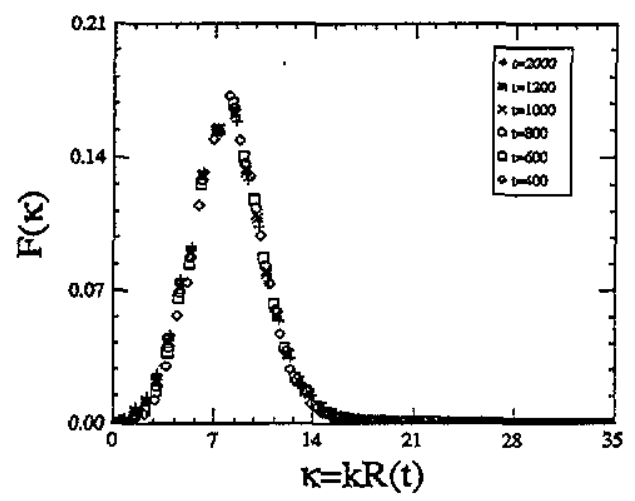

Figure 12. Dynamic scaling of model $D$ in the intermediate to late times $(t=400 \mathrm{up}$ to $t=2000$ ), for $\rho_{0}=0.17$, the wave numbers are from $k=0$ to $k=\pi$. Note that the curves oollapse on a single curve.

where the scaled wave number $x=k R(t)$, and $d$ is the dimension of the system. Figure 11 shows the scaled pair correlation function in the case of model $D$ and the scaled structure factor is shown in figure 12 .

For models $\mathrm{A}$ and $\mathrm{C}$, the structure factor corresponding to the order parameter exhibits a peak at $k=0$. This implies that, although the domains are not correlated in the same way as found for model $D$, they can still be characterized in terms of an average size. Figure 13 gives the scaled structure factor corresponding to model $\mathrm{C}$ and good scaling is again observed.

While non-zero values of $\rho_{0}$ lead to the slow dynamics mentioned above, we obtain a $t^{1 / 3}$ growth law for $\rho_{0}=0$ for conserved order parameters as found for spinodal decomposition $[15,16]$. A crossover between the two distinct behaviours is therefore expected. We also expect the equilibrium domain size to be proportional to $1 / \rho_{0}$ as observed in bicontinuous microemulsions [10], since the surfactants mostly accumulate at the interfaces. This implies that the total length of the interfaces is proportional to $\rho_{0}$. These considerations suggest the following crossover scaling

$$
R(t) t^{-1 / 3}=f\left(\rho^{3} t\right)
$$




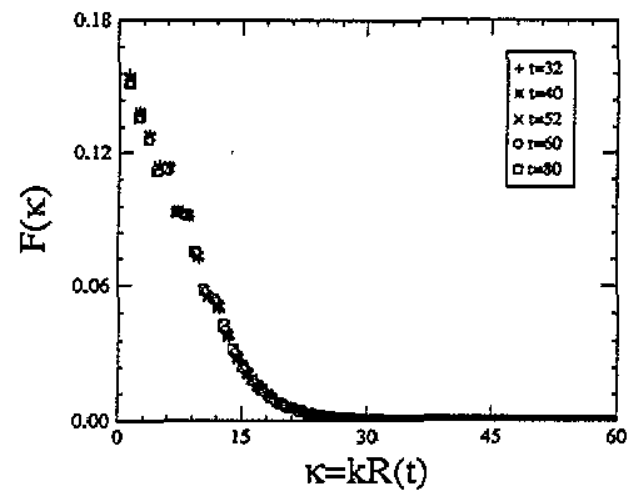

Figure 13. Dynamic scaling of model $C$ for $\rho_{0}=0.15$ in the case of model $C$ for times ranging from $t=32$ up to $t=60$, and wave number from $k=0$ to $k=\pi$.

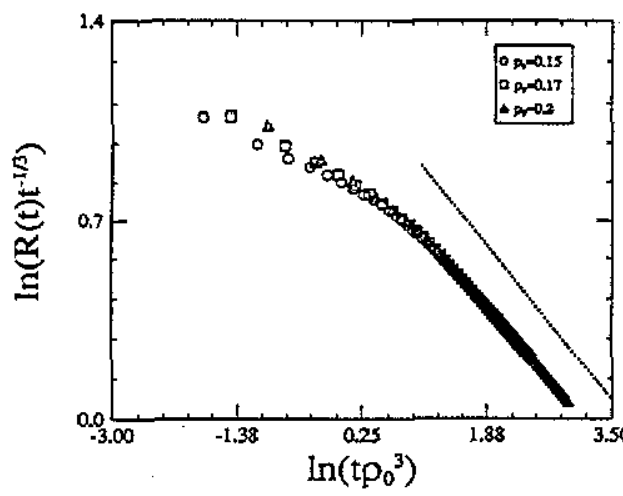

Figure 14. Crossover function for the average domain size in model $D$ for different values of $\rho_{0}$. The straight line has a slope of $-\frac{1}{3}$.

The function $f(\tau)$ is equal to a constant for $\tau=0$ and is proportional to $\tau^{-1 / 3}$ for large $\tau$. Our data for $\rho_{0}$ ranging from 0.15 to 0.20 are consistent with this crossover form, as shown in figure 14. However, a detailed confirmation of this crossover form needs more data for smaller values of $\rho_{0}$.

\section{Conclusion}

In this work, a Ginzburg-Landau model based on two local fields was used to examine the dynamics of microphase separation for a ternary mixture of two phase-separating components containing surfactants. Following Halperin et al [24], we examined models $A, B, C$ and $D$ corresponding to the four possible conservation laws for the two local fields of the model, while emphasizing model $\mathrm{D}$ where both order parameters are conserved. The domain growth was studied by monitoring the correlation function and the structure factor. We observed slow logarithmic-like dynamics at intermediate times for all models for non-zero values of the surfactant concentration. The slow dynamics was attributed to the accumulation of surfactants at the interfaces after their migration from the bulk. This drastically reduced the interfacial tension and thus the 
driving force. The average domain size was observed to decrease as the surfactant concentration increased. A self-similar behaviour was found from dynamic scaling of the correlation function and from the structure factor at intermediate times. Although the average domain size remained finite, it was found to be much larger than the width of interfaces and therefore was taken as the dominant length scale. Recently, Yao and Laradji [29] developed a theory for the dynamics of phase separation in the presence of surfactants when the concentration of water and oil are quite different and found similar results.

Our results are in qualitative agreement with the recent simulations of Kawakatsu and Kawasaki [30] on a hybrid model where water and oil are treated in a coarsegrained manner, whereas surfactants are treated microscopically using Newtonian laws; they also found slowing down in the domain growth. Finally, we do not expect our results to be qualitatively different in three dimensions, i.e., we expect approximate scaling and anomalously slow growth to be general features of the dynamics. This conjecture is in the process of being tested.

\section{Acknowledgments}

This work was supported by the Natural Sciences and Engineering Research Council of Canada, and les Fonds pour la Formation des Chercheurs et l'Aide à la Recherche de la Province de Québec.

\section{References}

[1] Witten T A 1990 Phys. Today 21 July

[2] Meunier J, Langevin D and Boccara N (ed) 1987 Physics of Amphiphilic Layers Part VI (Berlin: Springer) and references therein

[3] Kotlarchyk M, Chen S-H, Huang J S and Kim M W 1984 Phys. Rev. Lett. 53941

[4] Auvray L, Cotton J P, Ober R and Taupin C 1984 J. Physique 45913

Auvray L, Cotton J P, Ober R and Thupin C 1986 Physica B 136281

[5] Alba-Simionesco C, Teixeira J and Angell C A $1989 \mathrm{~J}$. Chem. Phys. 91395

[6] Wang Z-G and Safran S A $1990 \mathrm{~J}$. Physique 51185

Wang Z-G and Safran S A 1991 J. Chern. Phys. 94679

[7] Holyst R and Schick $1992 \mathrm{~J}$. Chem. Phys. 967728

[8] Wheeler J C and Widom B $1968 \mathrm{~J}$. Am. Chem. Soc. 903064

[9] Gompper G and Schick M 1989 Phys. Rev. Lett 62 1647; 1990 Phys. Rev. A 42, 2137; 1990 Phys. Rev. B 419148

[10] Laradji M, Guo H, Grant M and Zuckermann M J 1991 Phys. Rev. A 448184

[11] Laradji M, Guo H, Grant M and Zuckermann M J 1992 unpublished

[12] Chen K, Jayaprakash C., Pandit R and Wenzel W 1990 Phys. Rev. Lett. 652736

[13] Gunton J D, San Miguel M and Sahni P S 1983 Phase Transitions and Critical Phenomena ed C Domb and J L Lebowitz (London: Academic)

[14] Ohta T, Jasnow D and Kawasaki K 1982 Phys. Rev. Lett. 491223

[15] Rogers T M, Elder K R and Desai R C 1988 Phys. Rev. B 379638

[16] Roland C and Grant M 1988 Phys. Rev. Lett. 60 2657; 1989 Phys. Rev. B 3911971

[17] Grest G S and Srolovitz D J 1985 Phys. Rev. B 323014

Srolovitz D and Grest G 1985 Phys. Rev. B 323021

[18] Chowdlury D, Grant M and Gunton J D 1987 Phys. Rev. B 356792

[19] Villain J 1984 Phys. Rev. Lett. 521543

[20] Grant M and Gunton J D 1987 Phys. Rev B 354922

[21] Orguz E, Chakrabarti A, Toral R and Gunton J D 1990 Phys. Rev. B 42704

[22] Fisher K M 1977 Physica 86-88 813 
[23] Grant M and Gunton J D 1984 Phys. Rev B 296266

[24] Halperin B I, Hohenberg P C and Ma S K 1974 Phys. Rev. B 10139

[25] Laradji M, Guo H, Grant $M$ and Zuckermann M 1991 J. Phys. A: Math. Gen. 24 L629

[26] Hohenberg P C and Halperin B I 1977 Rev. Mod Phys. 49435

[27] Elder K R, Morin B and Grant M 1991 Phys. Rev. A 446673

[28] Chakrabarti A, Collins J B and Gunton J D 1989 Phys. Rev. B 386894

[29] Yao J H and Laradji M 1992 Phys. Rev. A submitted

[30] Kawakatsu T and Kawasaki K 1990 Physica A 167 690; 1991 J. Colloid. Interface Sci 145 413; 1991

J. Colloid. Interface Sci. 145420 